\title{
A post cytomegalovirus Guillain-Barré syndrome, with atypical presentation, associated with arachnoiditis in a 10-month-old girl
}

\author{
Nina Goldman', Jean-Christophe Cuvellier' ${ }^{1 *}$, Gustavo Soto-Ares ${ }^{2}$ and Louis Vallée ${ }^{1}$ \\ ${ }^{1}$ Division of Paediatric Neurology, Department of Paediatrics, Lille Faculty of Medicine and Children's Hospital, France \\ ${ }^{2}$ Department of Neuroradiology, University Hospital Roger Salengro, Lille Faculty of Medicine and Children's Hospital, France
}

\begin{abstract}
We present the case of a 10-month-old girl with Guillain-Barré syndrome who was diagnosed in a late stage of the disease. The initial presentation was atypical both clinically (severe back pain and meningismus) and radiologically (diffuse leptomeningeal enhancement spreading from the posterior fossa to the cauda equina). This led to a strong suspicion of carcinomatous meningitis or tuberculous spinal arachnoiditis. Considering absence of deep tendon reflexes, the patient was finally diagnosed with Guillain-Barré syndrome associated with cytomegalovirus infection. Her condition improved following intravenous immunoglobulin treatment. To our knowledge, whereas enhancement of the cauda equine roots represents the neuroradiological hallmark of Guillain-Barré syndrome, such a diffuse leptomeningeal enhancement has never been reported in association with Guillain-Barré syndrome. Paediatricians should consider Guillain-Barré syndrome in their differential diagnosis not only when faced with a child who has back pain and meningismus, but also in case of diffuse spinal arachnoiditis.
\end{abstract}

Abbreviations: GBS: Guillain-Barré syndrome; DTR: Deep Tendon Reflexes; MRI: Magnetic Resonance Imaging; CSF: Cerebrospinal Fluid; CMV: Cytomegalovirus

\section{Introduction}

Guillain-Barré syndrome (GBS) is an acute inflammatory demyelinating polyradiculoneuropathy characterized by ascending symmetrical weakness and loss of deep tendon reflexes (DTR), preceded by sensory abnormalities. Albuminocytologic dissociation is the hallmark of GBS.

We present a case of atypical GBS who was diagnosed in a late stage of the disease, due to numerous pitfalls, both clinical and radiological, besides the young age of the patient.

\section{Case report}

A 10-month-old girl, first child of consanguineous parents of Maghreb origin had no other remarkable medical history. She presented with a one-month history of unexplained crying, followed by nasopharyngeal infection without fever. A few days later, she refused to sit and ultimately to stand. The patient progressively developed dysphonia and intermittent choking on liquids. Recent history revealed no travel, walk in forest, vaccine or infection in the relatives. Growth curve showed weight stagnation from 5-6 months onwards. On clinical exam, the patient appeared extremely painful. Contrasting with the refusal to stand and sit, motility and cranial nerves appeared normal, but there was resistance to passive neck flexion and DTR were absent. There was no sphincter disturbance. Brain and spinal magnetic resonance imaging (MRI) showed diffuse leptomeningeal enhancement spreading from the posterior fossa to the cauda equina (Figures 1 and 2). Cerebrospinal fluid (CSF) examination revealed $4 \mathrm{WBC} / \mathrm{mm}^{3}$, total protein of $0.58 \mathrm{~g} / \mathrm{dL}$ and a glucose level of $97 \mathrm{mg} / \mathrm{dL}$.

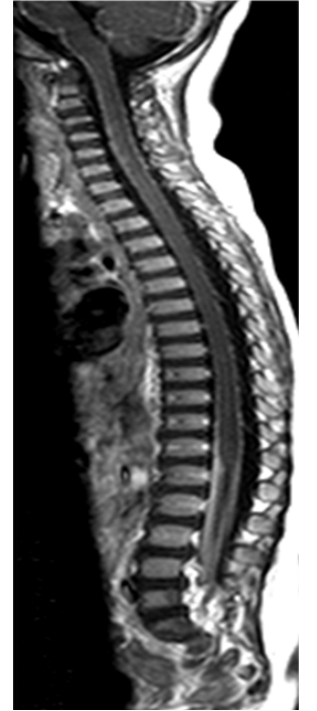

Figure 1. T1 sagittal weighted imaging after gadolinium showing an extended pial lining and arachnoid enhancement associated due to the presence of arachnoiditis

${ }^{\star}$ Correspondence to: Jean-Christophe CUVELLIER, Division of Paediatric Neurology, Department of Paediatrics, Lille Faculty of Medicine and Children's Hospital, 59037 Lille cedex, France, Tel: 330-320-444-057, Fax: 330-320-445393, E-mail: Jean-Christophe.CUVELLIER@CHRU-LILLE.FR

Key words: arachnoiditis, cytomegalovirus, Guillain-Barré syndrome, magnetic resonance imaging, pain

Received: August 03, 2018; Accepted: August 22, 2018; Published: August 25, 2018 


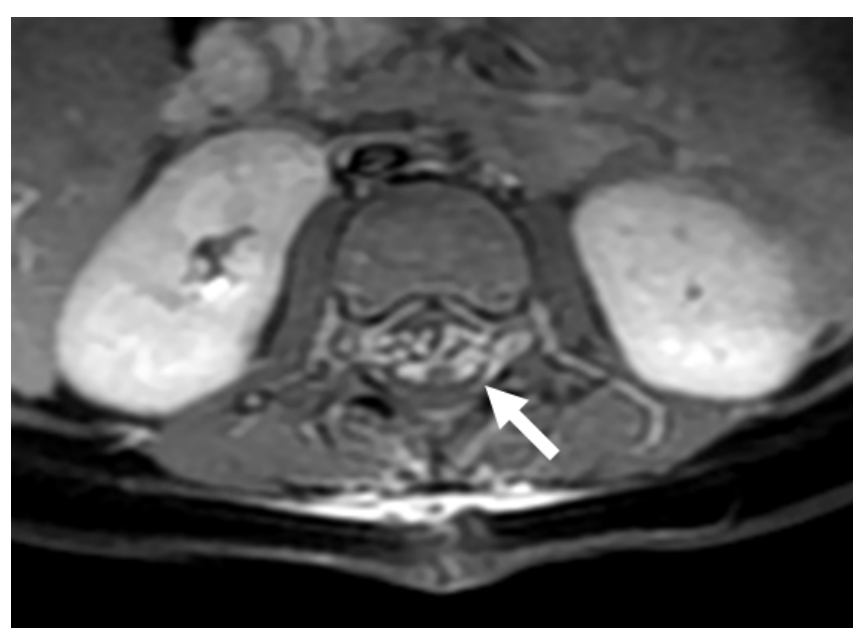

Figure 2. T1 axial weighted imaging after gadolinium showing thickness of both anterior and posterior nerves roots of the cauda equina due to the presence of arachnoiditis (arrow)

The initial neurological examination was challenging because of deep pain and meningeal signs, combined with diffuse leptomeningeal enhancement. This led us to suspect strongly carcinomatous meningitis or tuberculous spinal arachnoiditis. Differential diagnoses including infections, inflammatory and metabolic disease were ruled out by appropriate laboratory studies. Complete blood count showed thrombocytosis $\left(508000 / \mathrm{mm}^{3}\right)$. Laboratory screen was normal for CRP, procalcitonin, electrolytes, liver and renal function tests, lactate, LDH, acid uric erythrocyte sedimentation rate, electrophoresis of plasma protein, haemostasis, whereas serum vitamin $\mathrm{D}(14 \mathrm{ng} / \mathrm{ml})$ and ferritin (13 ng / ml) concentrations were low. CSF analysis (IgG index and oligoclonal bands, myelin-basic protein and Neuron Specific Enolase, cultures and serology, when appropriate, for Herpes simplex virus, Epstein barr virus, enterovirus, parvovirus, rubella, campylobacter, Lyme disease and mycoplasma) showed no abnormality. There was no bacterial or viral growth in the blood, urine, stool, or CSF. PCR (CMV, HSV, enterovirus) as well as search for cryptococcal in the CSF were negative. Serological testing showed that cytomegalovirus (CMV) IgM and IgG levels were elevated to $93.2 \mathrm{U} / \mathrm{mL}$ (normal level, $<22 \mathrm{U} / \mathrm{mL}$ ) and $24.2 \mathrm{U} / \mathrm{mL}$ (normal level, $<14 \mathrm{U} / \mathrm{mL}$ ), respectively. Ophthalmological examination including fundoscopy was normal. Meningeal biopsy disclosed spinal arachnoiditis without malignancy. Nerve conduction studies performed 3 weeks following admission demonstrated multifocal demyelination with prolonged distal motor latencies and F-wave latencies in the median and ulnar nerves (asymmetrically prolonged distal latencies, F-wave minimal latencies and H-reflex latencies.). The patient's history, physical examination, and laboratory findings were consistent with a diagnosis of GBS associated with CMV infection. She was treated with $2 \mathrm{~g} / \mathrm{kg}$ of intravenous immunoglobulin over 2 days. The patient's pain level decreased gradually whereas there was a marked functional improvement with a recovery of the sitting, getting on all fours and standing in a few days. Repeat CSF examination was remarkable for a decrease of protein level to of $172 \mathrm{mg} / \mathrm{dL}$. She was discharged from the hospital with gabapentin, iron and vitamin $\mathrm{D}$, and intense motor rehabilitation. Two months later she manifested complete recovery with normal clinical examination including DTR. The patient did not attend the follow-up visit months later so that it was not possible to assay nerve conduction studies.

\section{Discussion}

We present a case of GBS who was diagnosed in a late stage of the disease. We were misled by the spinal arachnoiditis on the one hand and because we discarded the important clue consisting in the absence of DTR, on the other hand. Meningeal signs and elevated CSF protein were also attributed to arachnoiditis, contributing to diagnostic delay. If meningismus and prominent muscle pain causing irritability, alone or in combination have previously been reported in paediatric GBS, causing early diagnostic confusion, our case report is noteworthy for the presence and importance of the arachnoiditis [1,2]. In older children and adults arachnoiditis is typically responsible for chronic severe back and/or lower extremity/leg pain. Arachnoiditis may be associated with leptomeningeal infections of the spine, CNS neoplasms that spread into the subarachnoid space or common systemic malignant diseases that result in leptomeningeal metastases [3]. MRI findings include diffuse enhancement of nerve roots and of the leptomeninges [3].

Spinal MRI with gadolinium has recently been advocated by some authors as an additional tool to aid clinicians in establishing GBS diagnosis early in the course of the disease, notably when other diagnostic tests may still produce normal results. The two most common patterns in typical cases of GBS include enhancement of the nerve roots diffusely, or only of the ventral spinal nerve roots $[4,5]$.

We were unable to find GBS cases with such a diffuse leptomeningeal enhancement similar to our patient in the literature. In adults, there are several reports of diffuse enhancement of the cauda equina in GBS and of diffuse enhancement of lumbar nerve roots and of the pial lining of the conus medullaris in AIDS-related polyradiculopathy attributable to CMV. Post-operative cases or cases associated with medullary procedures such as myelography have also been reported [6-9]. Echaniz-Laguna and Philippi [10] have reported on a 54-year-old man presenting with chronic inflammatory demyelinating polyradiculoneuropathy causing spinal cord compression. Contrast-enhanced T1-weighted MRI studies demonstrated massive hypertrophy of cervical nerve roots causing cervical spinal cord compression and major hypertrophy of brachial plexi [10].

In our patient, we can wonder what were the respective contributions of the CMV, the young age of the patient, the ethnic background, the consanguinity and the targets of the antiganglioside antibodies. Enhancement of the nerve roots with gadolinium occurs when inflammation produces disruption in the blood-nerve barrier, leading to transudation of serum proteins into CSF. CMV IgM antibodies are presents in $8-15 \%$ of patients at the onset of GBS [11]. The presence of CMV DNA has been demonstrated in almost one-third of serum and cerebrospinal fluid samples from GBS patients who were positive for $\mathrm{CMV}$-specific antibodies at the onset of the neurological disease. GBS patients with CMV-specific IgM antibodies have clinical profiles that differ from those without serological evidence of a recent CMV infection: they are younger, and have more severe sensory abnormalities, more frequent facial weakness, and a more frequent severe general weakness that required artificial ventilation, with a consequent delayed recovery [12].

\section{Conclusion}

Clinicians need to be aware of atypical presentations of GBS. A careful neurologic exam including strength and evaluation of reflexes is imperative when considering GBS in the differential. The lesson to be learned from this case report is the need to not blindly rely on paraclinical results.

\section{References}

1. Nguyen DK, Agenarioti-Bélanger S, Vanasse M (1999) Pain and the Guillain-Barré syndrome in children under 6 years old. J Pediatr 134: 773-776. [Crossref]

2. Bradshaw DY, Jones HR (2001) Pseudomeningoencephalitic presentation of pediatric Guillain-Barré syndrome. J Child Neurol 16: 505-508. [Crossref] 
3. Georgy BA, Snow RD, Hesselink JR (1996) MR imaging of spinal nerve roots: techniques, enhancement patterns, and imaging findings. AJR Am J Roentgenol 166: 173-179. [Crossref]

4. Coşkun A, Kumandaş S, Paç A, Karahan OI, Guleç M, et al. (2003) Childhood Guillain-Barré syndrome. MR imaging in diagnosis and follow-up. Acta Radiol 44: 230-235. [Crossref]

5. Mulkey SB, Glasier CM, El-Nabbout B, Walters WD, Ionita C, et al. (2010) Nerve root enhancement on spinal MRI in pediatric Guillain-Barré syndrome. Pediatr Neurol 43: 263-269. [Crossref]

6. Bazan C 3rd, Jackson C, Jinkins JR. Barohn AJ (1991) Gadolinium-enhanced MRI in a case of cytomegalovirus polyradiculopathy. Neurology 41: 1522-1523. [Crossref]

7. Talpos D, Tien AD, Hesselink JR (1991) Magnetic resonance imaging of AIDS related polyradiculopathy. Neurology 41: 1995-1997. [Crossref]
8. Kelley RE, Daroff RB, Sheremata WA, McCormick JR (1980) Unusual effects of metrizamide lumbar myelography. Constellation of aseptic meningitis, arachnoiditis, communicating hydrocephalus, and Guillaine-Barré syndrome. Arch Neurol 37: 588589. [Crossref]

9. Rosenberg SK, Stacey BR (1996) Postoperative Guillain-Barré syndrome, arachnoiditis, and epidural analgesia. Reg Anesth 21: 486-489. [Crossref]

10. Echaniz-Laguna A, Philippi N (2009) Teaching neuroimages: Chronic inflammatory demyelinating polyradiculoneuropathy causing spinal cord compression. Neurology 72: e121. [Crossref]

11. Steininger C (2007) Clinical relevance of cytomegalovirus infection in patients with disorders of the immune system. Clin Microbiol Infect 13: 953-963. [Crossref]

12. Visser LH, van der Meché FG, Meulstee J, Rothbarth PP, Jacobs BC, et al (1996) Cytomegalovirus infection and Guillain-Barré syndrome: the clinical, electrophysiologic, and prognostic features. Dutch Guillain-Barré Study Group. Neurology 47: 668-673. [Crossref]

Copyright: $(92018$ Goldman N. This is an open-access article distributed under the terms of the Creative Commons Attribution License, which permits unrestricted use, distribution, and reproduction in any medium, provided the original author and source are credited. 